\title{
Uso de compostos orgânicos no plantio do cafeeiro conilon
}

\author{
Luiz Augusto Lopes Serrano ${ }^{1}$, Victor Maurício da Silva², Edegar Antônio Formentini ${ }^{3}$
}

\section{RESUMO}

O cafeeiro conilon (Coffea canephora, Pierre ex Froenhner) apresenta elevado potencial produtivo e alta exigência nutricional. Entretanto, devido ao aumento nos custos dos fertilizantes minerais, o fornecimento das quantidades recomendadas de nutrientes vem se tornando limitante para os produtores rurais. O objetivo deste trabalho foi avaliar a utilização de compostos orgânicos no plantio do cafeeiro conilon, com o intuito de substituir as adubações de cobertura com $\mathrm{N}$ e K recomendadas na fase de pós-plantio. O experimento foi realizado em casa-de-vegetação, em Linhares-ES, sob delineamento experimental inteiramente casualizado, em esquema fatorial (2x5), com cinco repetições. Foram avaliadas cinco doses de dois compostos orgânicos misturados ao solo. Cada parcela experimental foi constituída por um vaso de 12,8 L, que recebeu uma planta. Os compostos constituíram-se da mistura de esterco bovino curtido e capim-elefante (C1), e de cama-de-frango e capim-elefante (C2); ambos na proporção 1:4. As doses utilizadas foram $0 ; 0,853 ; 1,706 ; 2,559$ e 3,412 kg/vaso do C1; e $0 ; 0,758 ; 1,516 ; 2,274$ e 3,032 kg/vaso do C2. Tanto o tipo do composto orgânico como as doses aplicadas interferiram no crescimento e nos teores foliares de macronutrientes. As doses que proporcionaram os maiores acúmulos de matéria seca total foram de 1,623 kg/vaso $\approx 8,115 \mathrm{~kg} / \mathrm{cova}$ de 64 L) do $\mathrm{C} 1$ e $1,733 \mathrm{~kg} / \mathrm{vaso}(\approx 8,665 \mathrm{~kg} /$ cova de $64 \mathrm{~L})$ do C2. Nessas doses, os teores foliares de $\mathrm{N}$ e K estiveram acima da faixa de suficiência. Concluiu-se que os compostos orgânicos podem substituir ou reduzir as adubações de cobertura com N e K na fase de pós-plantio do cafeeiro conilon.

Palavras-chave: Coffea canephora Pierre ex Froenhner, esterco bovino, cama-de-frango, Pennisetum purpureum Schumach.

\section{ABSTRACT}

\section{Use of organics compounds in the planting coffee conilon trees}

Conilon coffee trees (Coffea canephora Pierre ex Froenhner) show high yield potential and nutritional requirements. However, due to increase mineral fertilizers costs, the supply of the recommended amounts to be applied is an obstacle for farmers. Thus, the objective of this work was to evaluate the use of two organic compounds in planting of Coffea canephora trees, in order to replace the $\mathrm{N}$ and $\mathrm{K}$ mineral fertilizer recommended for phase post-planting. The research was realized in greenhouse, in Linhares-ES, Brazil. The experimental design was a completely randomized in a factorial scheme $(2 \times 5)$, with five replications. Each experimental plot consisted of one plant per container (12.8 L). It was evaluated five doses of two organic compounds that were mixed to soil used with substrate. The organic compound 1 (C1) was composed by the mixture of cattle manure and Pennisetum purpureum, and the organic compound 2 (C2) was composed by the mixture of poultry litter and Pennisetum purpureum; both in proportion 1:4. The doses of $\mathrm{C} 1$ were 0 ; $0.853 ; 1.706 ; 2.559$ and $3.412 \mathrm{~kg} /$ container; and the doses of C2 were $0 ; 0.758 ; 1.516 ; 2.274$ e $3.032 \mathrm{~kg} / \mathrm{container}$. The results showed that both the type of organic compound and the amount applied, interfered on plants growth and in

Recebido para publicação em agosto de 2009 e aprovado em agosto de 2010

${ }^{1}$ Engenheiro-Agrônomo, Doutor. Empresa Brasileira de Pesquisa Agropecuária, EMBRAPA-CNPAT, Rua Dra . Sara Mesquita, 2.270, 60511-110, Fortaleza, CE, Brasil. serrano@cnpat.embrapa.br.

${ }^{2}$ Biólogo. Mestrando da Universidade Federal do Espírito Santo, CCAUFES, Departamento de Produção Vegetal, Caixa Postal 16, 29500-000, Alegre, ES, Brasil. victormauricio@bol.com.br.

${ }^{3}$ Engenheiro-Agrônomo. Instituto Capixaba de Pesquisa, Assistência Técnica e Extensão Rural - INCAPER, Rua Afonso Sarlo, 160, 29052-010, Vitória, ES, Brasil. formentini@incaper.es.gov.br. 
foliar nutrient levels. The highest doses of both compounds retarded the plants development. The doses that provided better dry matter accumulation were $1.623 \mathrm{~kg} /$ container $(\approx 8.115 \mathrm{~kg} / \mathrm{hole})$ of C1 e $1.733 \mathrm{~kg} /$ container $(\approx 8.665 \mathrm{~kg} / \mathrm{hole})$ of $\mathrm{C} 2$. At these same doses, the plants showed foliar levels of $\mathrm{N}$ and $\mathrm{K}$ above the sufficiency range. This way, the organic compounds may be an alternative to replace or reduce the $\mathrm{N}$ and $\mathrm{K}$ mineral fertilizer recommended for postplanting Coffea canephora trees.

Key words: Coffea canephora Pierre ex Froehner, cattle manure; poultry litter; Pennisetum purpureum Schumach.

\section{INTRODUÇÃO}

A cafeicultura desempenha função relevante no desenvolvimento social e econômico do Brasil, garantindo a geração de empregos e tributos, contribuindo significativamente para a formação da receita brasileira. Com relação à produção, a previsão para a safra 2009/2010 é de que o Brasil produza 11,27 milhões de sacas beneficiadas de café conilon (Coffea canephora), o que representa 23,4\% da produção nacional de café. O Estado do Espírito Santo é o maior produtor nacional deste café, respondendo por $65,3 \%$ da produção (CONAB, 2010).

Segundo Bragança et al. (2007), os clones de café conilon oriundos de seleções feitas em programas de melhoramento genético apresentam um potencial produtivo elevado. Dessa forma, esses genótipos são de alta exigência nutricional e acumulam grande quantidade de nutrientes.

Informações sobre os teores foliares de nutrientes em mudas de cafeeiro conilon são escassas, enquanto que para plantas adultas Bragança et al. (2007) sugerem como faixas de suficiência de macronutrientes os teores foliares de 2,90 a 3,20 dag $\mathrm{kg}^{-1}$ de N; 0,12 e 0,16 dag kg ${ }^{-1}$ de P; 2,00 a 2,50 dag kg-1 de K; 1,00 a 1,50 dag $\mathrm{kg}^{-1}$ de $\mathrm{Ca}$; 0,35 a 0,40 dag $\mathrm{kg}^{-1}$ de $\mathrm{Mg}$; e 0,20 a $0,25 \mathrm{dag} \mathrm{kg}^{-1}$ de $\mathrm{S}$. Ao estabelecer as normas DRIS para o cafeeiro conilon, Partelli et al. (2006) obteve, respectivamente, as seguintes médias de teores foliares para lavouras de alta produtividade nos sistemas orgânico e convencional: 2,76 e 2,64 dag kg-1 de $\mathrm{N} ; 0,16$ e 0,14 dag kg${ }^{-1}$ de P; 1,67 a 1,85 dag $\mathrm{kg}^{-1}$ de K; 1,35 e 1,16 dag kg-1 de Ca; 0,35 e 0,36 dag $\mathrm{kg}^{-1}$ de $\mathrm{Mg}$; e 0,21 e $0,18 \mathrm{~g} \mathrm{~kg}^{-1}$ de $\mathrm{S}$.

Para suprir a exigência elevada em nutrientes, a reposição destes no solo é feita através da aplicação de adubos minerais. Entretanto, devido a dependência internacional de fertilizantes e do seu alto custo, o fornecimento das quantidades corretas de nutrientes é um entrave para os produtores rurais. Segundo a Associação Nacional para Difusão de Adubos (ANDA, 2009), os custos das principais formulações subiram em média $110 \%$ em 2008. O Brasil importa cerca de $70 \%$ do nitrogênio que consome, e produz apenas $10 \%$ do potássio que utiliza. No caso do fósforo, não há tantas limitações, pois o país possui jazidas (rochas) disponíveis, o que garante uma produção suficiente.

Diante do exposto, o uso racional dos insumos nos sistemas convencional ou orgânico torna-se imprescindível para o sucesso da atividade agrícola. Uma alternativa para substituir ou reduzir as quantidades de adubos minerais utilizados na agricultura é o uso de fontes orgânicas de nutrientes, que podem ser conseguidas na própria propriedade rural, como palhada de gramíneas e ou leguminosas, casca de café oriunda do beneficiamento deste, estercos e dejetos de animais, dentre outros; e que podem ser compostados.

A eficiência na aplicação incorporada de adubo orgânico no cafeeiro arábica (Coffea arabica L.) já foi comprovada por Cervellini \& Igue (1994) e por Cervellini et al. (1995). Deste modo, o objetivo do trabalho foi avaliar a utilização de dois compostos orgânicos, provindos de materiais de fácil aquisição pelos produtores rurais, como adubo de plantio na cultura do cafeeiro conilon, com o intuito de substituir as adubações nitrogenada e potássica recomendadas para a fase pós-plantio.

\section{MATERIAL E MÉTODOS}

O experimento foi realizado em casa-de-vegetação localizada na Fazenda Experimental do INCAPER, em Linhares-ES (19²5'03" S, 4004'50" O e 17 m), sob delineamento experimental inteiramente casualizado (DIC), em esquema fatorial ( $2 \times 5)$, com cinco repetições. Cada repetição foi composta por uma planta por vaso.

Foram avaliadas cinco doses de dois compostos orgânicos misturados a $64 \mathrm{~L}$ de solo. Após a mistura das respectivas doses dos compostos ao solo, o substrato obtido foi distribuído em cinco vasos (repetições), totalizando um volume de $12,8 \mathrm{~L}$ de substrato por vaso.

O primeiro composto orgânico $(\mathrm{C} 1)$ foi preparado com a mistura de esterco bovino curtido e capim-elefante (Pennisetum purpureum Schumach), e o segundo composto orgânico (C2) foi preparado pela mistura de cama-de-frango e capim-elefante, ambos na proporção 1:4 (v:v).

Para o processo de compostagem, a montagem das leiras foi realizada no mesmo dia do corte do capim, em um 
pátio cimentado sob cobertura. Inicialmente houve o empilhamento do resíduo vegetal por camada de, no máximo, $30 \mathrm{~cm}$ de altura, seguida de uma fina camada de 3 a 5 $\mathrm{cm}$ de esterco bovino curtido (C1) ou de cama-de-frango (C2). O primeiro reviramento das leiras foi realizado sete dias depois de montadas as leiras e os demais quinzenalmente, totalizando quatro reviramentos. Para garantir um umedecimento uniforme, optou-se por irrigar as leiras antes dos reviramentos.

Após 90 dias da montagem das leiras, através das características físicas observadas (coloração, temperatura, granulometria, entre outras), foi constatado que os compostos estavam adequados para utilização. Em seguida, amostras dos compostos foram submetidas às análises químicas realizadas no Laboratório de Análises de Solos do INCAPER/CRDR-CS.

De acordo com os resultados das análises químicas, constatou-se que o $\mathrm{C} 1$ continha $33 \%$ de umidade, $38 \mathrm{dag}$ $\mathrm{kg}^{-1}$ de matéria orgânica, relação C:N de 14:1 e teores totais na matéria seca de $1,60 \mathrm{dag} \mathrm{kg}^{-1} \mathrm{de} \mathrm{N}, 0,44 \mathrm{dag} \mathrm{kg}^{-1} \mathrm{de}$ P, 2,47 dag kg-1 de K, 0,93 dag kg-1 de Ca, 0,23 dag $\mathrm{kg}^{-1}$ de Mg, 0,20 dag kg-1 de S, $100 \mathrm{mg} \mathrm{kg}^{-1}$ de Zn, $3.287 \mathrm{mg} \mathrm{kg}^{-1}$ de $\mathrm{Fe}, 444 \mathrm{mg} \mathrm{kg}^{-1}$ de $\mathrm{Mn}, 14 \mathrm{mg} \mathrm{kg}^{-1}$ de Cu e $9 \mathrm{mg} \mathrm{kg}^{-1}$ de B. Já o C2 continha $33 \%$ de umidade, $58 \mathrm{dag} \mathrm{kg}^{-1}$ de matéria orgânica, relação C:N de 19:1 e teores totais na matéria seca de 1,80 dag kg ${ }^{-1}$ de N, 0,77 dag kg-1 de P, 2,80 dag kg${ }^{1}$ de K, 5,21 dag kg-1 de Ca, 0,17 dag kg-1 de Mg, 0,28 dag $\mathrm{kg}^{-1}$ de S, $275 \mathrm{mg} \mathrm{kg}^{-1}$ de Zn, $1.870 \mathrm{mg} \mathrm{kg}^{-1}$ de Fe, $327 \mathrm{mg}$ $\mathrm{kg}^{-1}$ de Mn, $100 \mathrm{mg} \mathrm{kg}^{-1}$ de Cu e $29 \mathrm{mg} \mathrm{kg}^{-1}$ de B.

Baseado nas análises químicas dos compostos orgânicos (teor de N) e nas recomendações de Guimarães et al. (1999) e Prezotti et al. (2007) para a adubação de pósplantio em cobertura para a cultura do cafeeiro - que consiste na aplicação de $\mathrm{N}$ a partir do pegamento das mudas até o final do período chuvoso - optou-se pela utilização das seguintes doses dos compostos: 0 (D0); 0,853 (D1); 1,706 (D2); 2,559 (D3) e 3,412 (D4) kg/vaso do C1; e 0 (D0); 0,758 (D1); 1,516 (D2); 2,274 (D3) e 3,032 (D4) $\mathrm{kg} /$ vaso do C2.

Os resultados das análises granulométrica e química do solo utilizado no experimento mostraram que o mesmo apresentou textura franco arenosa; $\mathrm{pH} 6,0 ; 1,7 \mathrm{dag}$ $\mathrm{kg}^{-1}$ de matéria orgânica; $2 \mathrm{mg} \mathrm{dm}^{-3} \mathrm{de} \mathrm{P} ; 78 \mathrm{mg} \mathrm{dm}^{-3} \mathrm{de}$ $\mathrm{K} ; 1,6 \mathrm{cmol}_{\mathrm{c}} \mathrm{dm}^{-3} \mathrm{de} \mathrm{Ca} ; 0,4 \mathrm{cmol}_{\mathrm{c}} \mathrm{dm}^{-3} \mathrm{de} \mathrm{Mg} ; 0,0 \mathrm{cmol}_{\mathrm{c}}$ $\mathrm{dm}^{-3} \mathrm{de} \mathrm{Al} ; 1,7 \mathrm{cmol}_{\mathrm{c}} \mathrm{dm}^{-3} \mathrm{de} \mathrm{H}+\mathrm{Al} ; 2,2 \mathrm{cmol}_{\mathrm{c}} \mathrm{dm}^{-3} \mathrm{de}$ soma de bases (SB) e 56,4\% de saturação de bases (V). Com base na análise do solo, cada vaso recebeu 3,4 g de calcário dolomítico (PRNT 96\%) e 40 g de superfosfato simples.

Foram plantadas nos vasos mudas do cafeeiro conilon clone $12 \mathrm{~V}$, componente da variedade 'Vitória - INCAPER 8142 ', com 3 a 4 pares de folhas completamente desenvolvidas e apenas um ramo ortotrópico.
O plantio ocorreu no dia 03/10/2008. As plantas foram irrigadas diariamente, e houve a necessidade de aplicações de Bioneem ${ }^{\circledR}\left(1 \mathrm{ml} \mathrm{L}^{-1}\right)$, para o controle de cochonilhas, e de calda bordalesa, para o controle de Cercospora coffeicola, visto que as plantas dos tratamentos que não receberam os compostos apresentaram deficiência de N, o que favoreceu o surgimento da doença (Pozza et al., 2001).

Aos 30, 60, 90 e 120 dias após o plantio das mudas, foram realizadas avaliações do crescimento das plantas: altura das plantas (base do broto novo até a gema apical), diâmetro do caule (a $1 \mathrm{~cm}$ da base do broto novo) e o número de folhas completamente expandidas.

$\mathrm{Na}$ última avaliação, as plantas foram coletadas e foram avaliados o número de ramos plagiotrópicos, o volume da raiz e a área foliar. $\mathrm{O}$ volume da raiz foi mensurado através da imersão do sistema radicular em água contida numa proveta graduada e a área foliar através de um aparelho medidor de área foliar.

Em seguida, as plantas foram secas em estufa $\left(70^{\circ} \mathrm{C}\right.$ por 72 horas) com posterior determinação das massas das matérias secas das folhas, dos ramos (ortotrópicos + plagiotrópicos) e do sistema radicular. Por fim, prepararam-se amostras foliares de cada parcela experimental que foram encaminhadas para o Laboratório de Análises de Solos e Foliar do INCAPER/CRDR-NE para a quantificação dos teores foliares de macronutrientes $(\mathrm{N}$, P, K, Ca, Mg e S).

Os dados obtidos foram submetidos à análise de variância. Para verificar os efeitos dos compostos utilizados, as médias foram comparadas pelo teste de Tukey, e para verificar os efeitos das doses de cada composto, as médias foram ajustadas pela análise de regressão, ambos a $5 \%$.

\section{RESULTADOS E DISCUSSÃO}

Os resultados obtidos mostraram que tanto o tipo de composto orgânico quanto as suas doses interferiu no crescimento e nos teores foliares de macronutrientes das plantas de cafeeiro conilon.

Aos 30 dias após o plantio (DAP), não foram observadas diferenças no crescimento das plantas nos diferentes tratamentos (Tabela 1), fato que pode ser atribuído ao consumo de suas próprias reservas, visto que as mesmas eram oriundas de estacas, ou devido a sua pouca exigência nessa fase em relação à quantidade de nutrientes existente no substrato. Já aos 60 DAP, foi observado que as plantas que receberam a maior dose do $\mathrm{C} 1$ apresentavam crescimento inferior às demais, talvez pela quantidade excessiva de nutrientes para a fase. Aos 90 DAP, esta inferioridade no crescimento também foi observada nos tratamentos testemunhas (C1 D0 e C2 D0), fato este que ficou bem evidente aos 120 DAP. 
Aos 120 dias após o plantio observou-se que as plantas dos tratamentos testemunhas e do tratamento que recebeu a maior dose do $\mathrm{C} 1$ apresentaram os menores valores em todas as características de crescimento avaliadas (Tabelas 1 e 2).

Em Marilândia, ES, Bragança et al. (2010) avaliou o crescimento do cafeeiro conilon clone 02 , pertencente à variedade clonal 'EMCAPA 8111', e verificou que, aos 90 dias após o plantio no campo, realizado em outubro, as plantas apresentaram em média, 15,97 g de massa de matéria seca de folhas; $10,13 \mathrm{~g}$ de massa de matéria seca de ramos; 8,71 g de massa de matéria seca de sistema radicular e 34,81 gramas de massa de matéria seca total. Aos 180 dias após o plantio, as plantas apresentaram em média,

Tabela 1. Características de crescimento das mudas de cafeeiro conilon após o plantio em vasos preenchidos com substratos compostos pela mistura de solo e diferentes doses de dois compostos orgânicos ${ }^{(1)}$

\begin{tabular}{|c|c|c|c|c|}
\hline \multirow{2}{*}{ Tratamentos $^{(2)}$} & 30 dias & 60 dias & 90 dias & 120 dias \\
\hline & \multicolumn{3}{|c|}{ Altura das plantas (cm) } & \\
\hline $\mathrm{C} 1$ (D0) & $6,80 \mathrm{a}$ & $12,48 \mathrm{a}$ & $19,28 \mathrm{bc}$ & $21,10 \mathrm{~b}$ \\
\hline C1 (D1) & $7,20 \mathrm{a}$ & $12,76 \mathrm{a}$ & $25,50 \mathrm{a}$ & $31,80 \mathrm{a}$ \\
\hline $\mathrm{C} 1$ (D2) & $6,84 \mathrm{a}$ & $12,00 \mathrm{a}$ & $24,90 \mathrm{a}$ & $32,90 \mathrm{a}$ \\
\hline C1 (D3) & $6,06 \mathrm{a}$ & $10,68 \mathrm{ab}$ & $21,48 a b$ & 29,24 a \\
\hline C1 (D4) & $4,78 \mathrm{a}$ & $6,64 \mathrm{~b}$ & $13,90 \mathrm{c}$ & $20,12 b$ \\
\hline C2 (D0) & $7,46 \mathrm{a}$ & $11,64 \mathrm{ab}$ & $17,00 \mathrm{bc}$ & $20,40 \mathrm{~b}$ \\
\hline $\mathrm{C} 2$ (D1) & $5,60 \mathrm{a}$ & $10,38 \mathrm{ab}$ & $24,92 \mathrm{a}$ & $33,60 \mathrm{a}$ \\
\hline $\mathrm{C} 2$ (D2) & $7,18 \mathrm{a}$ & $13,02 \mathrm{a}$ & $26,58 \mathrm{a}$ & $36,12 \mathrm{a}$ \\
\hline C2 (D3) & $6,88 \mathrm{a}$ & $11,84 \mathrm{a}$ & $21,88 \mathrm{ab}$ & $30,58 \mathrm{a}$ \\
\hline $\mathrm{C} 2$ (D4) & $8,10 \mathrm{a}$ & $13,16 \mathrm{a}$ & $21,36 \mathrm{ab}$ & 29,76 a \\
\hline Média Geral & 6,69 & 11,46 & 21,68 & 28,56 \\
\hline \multirow{2}{*}{$\mathrm{CV}(\%)$} & 22,95 & 21,27 & 16,31 & 13,18 \\
\hline & \multicolumn{3}{|c|}{ Diâmetro do caule (mm) } & \\
\hline C1 (D0) & $3,50 \mathrm{a}$ & $4,17 \mathrm{ab}$ & $5,36 a b$ & $6,23 \mathrm{bcd}$ \\
\hline C1 (D1) & $3,37 \mathrm{a}$ & $4,50 \mathrm{a}$ & $6,79 \mathrm{a}$ & $9,06 \mathrm{a}$ \\
\hline $\mathrm{C} 1$ (D2) & $3,04 \mathrm{a}$ & $4,00 \mathrm{ab}$ & $6,29 \mathrm{a}$ & $8,30 \mathrm{abc}$ \\
\hline C1 (D3) & $3,12 \mathrm{a}$ & $3,83 \mathrm{ab}$ & $5,99 \mathrm{a}$ & $8,52 \mathrm{a}$ \\
\hline C1 (D4) & $3,44 \mathrm{a}$ & $3,42 \mathrm{~b}$ & $4,09 \mathrm{~b}$ & $5,45 \mathrm{~d}$ \\
\hline C2 (D0) & $3,67 \mathrm{a}$ & $4,42 \mathrm{ab}$ & $5,46 \mathrm{ab}$ & $6,21 \mathrm{~cd}$ \\
\hline C2 (D1) & $3,37 \mathrm{a}$ & $4,10 \mathrm{ab}$ & $6,53 \mathrm{a}$ & $8,46 \mathrm{ab}$ \\
\hline $\mathrm{C} 2$ (D2) & $3,80 \mathrm{a}$ & $4,23 \mathrm{ab}$ & $6,52 \mathrm{a}$ & $8,77 \mathrm{a}$ \\
\hline C2 (D3) & $3,39 \mathrm{a}$ & $4,20 \mathrm{ab}$ & $5,80 \mathrm{ab}$ & $7,98 \mathrm{abc}$ \\
\hline C2 (D4) & $3,53 \mathrm{a}$ & $3,93 \mathrm{ab}$ & $5,90 \mathrm{ab}$ & $7,81 \mathrm{abc}$ \\
\hline Média geral & 3,42 & 4,08 & $\mathbf{5 , 8 7}$ & 7,68 \\
\hline \multirow[t]{2}{*}{ CV $(\%)$} & 11,61 & 11,94 & 14,55 & 13,78 \\
\hline & \multicolumn{3}{|c|}{ Número de folhas } & \\
\hline C1 (D0) & $11,40 \mathrm{a}$ & $12,80 \mathrm{a}$ & $27,00 \mathrm{bcd}$ & $34,40 \mathrm{~b}$ \\
\hline $\mathrm{C} 1$ (D1) & $10,80 \mathrm{a}$ & $17,40 \mathrm{a}$ & $49,60 \mathrm{a}$ & $94,80 \mathrm{a}$ \\
\hline $\mathrm{C} 1$ (D2) & $9,60 \mathrm{a}$ & $13,60 \mathrm{a}$ & $42,40 \mathrm{abc}$ & $87,20 \mathrm{a}$ \\
\hline C1 (D3) & $7,20 \mathrm{a}$ & $15,60 \mathrm{a}$ & $41,60 \mathrm{abc}$ & $83,40 \mathrm{a}$ \\
\hline C1 (D4) & $7,40 \mathrm{a}$ & $9,20 \mathrm{a}$ & $19,00 \mathrm{~d}$ & $38,60 \mathrm{~b}$ \\
\hline C2 (D0) & $8,80 \mathrm{a}$ & $13,60 \mathrm{a}$ & $24,00 \mathrm{~cd}$ & $35,80 \mathrm{~b}$ \\
\hline C2 (D1) & 8,60 a & $14,00 \mathrm{a}$ & $45,00 \mathrm{abc}$ & 86,20 a \\
\hline $\mathrm{C} 2$ (D2) & $9,60 \mathrm{a}$ & $14,80 \mathrm{a}$ & $50,60 \mathrm{a}$ & $96,60 \mathrm{a}$ \\
\hline C2 (D3) & $8,60 \mathrm{a}$ & $18,00 \mathrm{a}$ & $41,80 \mathrm{abc}$ & $85,20 \mathrm{a}$ \\
\hline C2 (D4) & $10,40 \mathrm{a}$ & $16,20 \mathrm{a}$ & $46,20 \mathrm{ab}$ & 83,60 a \\
\hline Média geral & 9,24 & 14,52 & 38,72 & 72,58 \\
\hline$\overline{\mathrm{CV}(\%)}$ & 31,12 & 31,48 & 26,71 & 22,14 \\
\hline
\end{tabular}

(1) Médias seguidas pela mesma letra nas colunas não diferem estatisticamente, pelo teste de Tukey, a 5\% de probabilidade.

(2) $\mathrm{C} 1$ : composto orgânico preparado pela mistura entre esterco bovino curtido e capim-elefante [0 (D0); 0,853 (D1); 1,706 (D2); 2,559 (D3) e 3,412 (D4) kg por vaso]; C2: composto orgânico preparado pela mistura entre cama-de-frango e capim-elefante [0 (D0); 0,758 (D1); 1,516 (D2); 2,274 (D3) e 3,032 (D4) kg por vaso]. 
18,06 g de massa de matéria seca de folhas; 22,97 g de massa de matéria seca de ramos; 12,23 g de massa de matéria seca de sistema radicular e 40,24 gramas de massa de matéria seca total.

Assim, as plantas do presente trabalho, exceto as dos tratamentos testemunhas e do tratamento que recebeu a maior dose do C1, avaliadas aos 120 DAP em casa-de-vegetação, apresentaram médias superiores de massas de matéria secas das folhas, dos ramos, do sistema radicular e total comparativamente às plantas cultivadas no campo por Bragança et al. (2010) (Tabela 2). Ressalta-se, porém, que em casa-de-vegetação as condições e os tratos culturais dados às plantas são mais eficientes e de mais fácil manejo se comparados ao cultivo em campo, sendo, então, considerado normal a superioridade observada. $\mathrm{O}$ fato importante é que as plantas que receberam as doses intermediárias dos dois compostos orgânicos apresentaram porte semelhante àquelas cultivadas em lavouras convencionais.

Para todas as características de crescimento avaliadas, foi observado um ajuste ao modelo quadrático concomitante ao aumento das doses dos compostos (Tabela 3). Estes resultados permitem inferir que doses elevadas destes compostos prejudicaram o crescimento das plantas de cafeeiro conilon. Andrade Neto et al. (1999) e Dias \& Melo (2009) também observaram efeito prejudicial de doses elevadas de materiais orgânicos no desenvolvimento de mudas de cafeeiro arábica.

De acordo com as respostas observadas foi possível estimar a dose adequada para a obtenção do valor máximo de cada característica de crescimento avaliada (Tabela
3). Com exceção do volume de raiz, para todas as outras características, as doses máximas do $\mathrm{C} 2$ foram superiores às do $\mathrm{C} 1$, fato contrário ao observado por Andrade Neto et al. (1999) ao utilizarem esterco de galinha e bovino, ambos puros e curtidos.

$\mathrm{Na}$ avaliação do crescimento de plantas geralmente leva-se em consideração apenas a altura, entretanto podem ocorrer distorções nos valores dessa característica devido ao excesso de N, ou seja, do crescimento da parte aérea em detrimento do sistema radicular. Logo, tornase importante considerar também a produção de matéria seca total da planta e também o diâmetro do caule (Marana et al., 2008). Assim, de acordo com os resultados deste trabalho, as doses que proporcionaram os valores máximos de altura das plantas, diâmetro de caule e matéria seca total foram de 1,645; 1,589 e 1,623 kg/vaso do $\mathrm{C} 1$, e 1,$747 ; 1,759$ e $1,733 \mathrm{~kg} /$ vaso do $\mathrm{C} 2$, respectivamente (Tabela 3 ).

Com relação aos teores foliares de nutrientes observados nas plantas de cafeeiro conilon deste experimento, ao serem comparados com os teores foliares de plantas adultas (Partelli et al., 2006 e Bragança et al., 2007), uma vez que há a escassez de dados referentes aos teores foliares de nutrientes em mudas, observou-se que as médias gerais obtidas para os teores foliares de N, P, K e S (Tabela 4) estão acima dos teores recomendados por estes autores.

Já os teores foliares de $\mathrm{N}$ nas plantas dos tratamentos testemunhas foram os menores (Tabela 4), fato que ocasionou sintomas visuais de deficiência de $\mathrm{N}$ nas plantas. Estas mesmas plantas apresentaram os maiores teores foliares de Ca, fato que corrobora a afirmação de Malavolta

Tabela 2: Características das mudas de cafeeiro conilon cultivadas em vasos preenchidos com substratos compostos pela mistura de solo e diferentes doses de dois compostos orgânicos, aos 120 dias após o plantio ${ }^{(1)}$

\begin{tabular}{lccccccc}
\hline Tratamentos $^{(2)}$ & $\mathbf{A F}\left(\mathbf{c m}^{2}\right)$ & VR $(\mathbf{m l})$ & NRP & MSF (g) & MSR (g) & MSSR (g) & MST (g) \\
\hline C1 (D0) & $1.074,80 \mathrm{~b}$ & $50,00 \mathrm{~b}$ & $5,40 \mathrm{c}$ & $7,32 \mathrm{c}$ & $3,32 \mathrm{bc}$ & $10,07 \mathrm{ab}$ & $20,71 \mathrm{bc}$ \\
C1 (D1) & $4.333,46 \mathrm{a}$ & $140,00 \mathrm{a}$ & $12,20 \mathrm{a}$ & $33,43 \mathrm{a}$ & $12,37 \mathrm{a}$ & $20,52 \mathrm{a}$ & $66,33 \mathrm{a}$ \\
C1 (D2) & $4.036,81 \mathrm{a}$ & $112,00 \mathrm{ab}$ & $10,80 \mathrm{ab}$ & $28,92 \mathrm{a}$ & $10,41 \mathrm{a}$ & $15,98 \mathrm{ab}$ & $55,31 \mathrm{ab}$ \\
C1 (D3) & $3.675,39 \mathrm{a}$ & $120,00 \mathrm{ab}$ & $10,80 \mathrm{ab}$ & $25,86 \mathrm{ab}$ & $9,27 \mathrm{ab}$ & $17,00 \mathrm{ab}$ & $52,13 \mathrm{abc}$ \\
C1 (D4) & $1.420,71 \mathrm{~b}$ & $30,00 \mathrm{~b}$ & $8,25 \mathrm{bc}$ & $9,25 \mathrm{bc}$ & $3,49 \mathrm{bc}$ & $4,42 \mathrm{~b}$ & $17,16 \mathrm{c}$ \\
C2 (D0) & $896,30 \mathrm{~b}$ & $46,00 \mathrm{~b}$ & $5,60 \mathrm{c}$ & $6,78 \mathrm{c}$ & $3,11 \mathrm{c}$ & $7,99 \mathrm{~b}$ & $17,88 \mathrm{c}$ \\
C2 (D1) & $4.274,18 \mathrm{a}$ & $122,00 \mathrm{ab}$ & $11,00 \mathrm{ab}$ & $33,01 \mathrm{a}$ & $11,67 \mathrm{a}$ & $19,49 \mathrm{a}$ & $64,17 \mathrm{a}$ \\
C2 (D2) & $5.112,73 \mathrm{a}$ & $128,00 \mathrm{a}$ & $12,20 \mathrm{a}$ & $39,65 \mathrm{a}$ & $13,89 \mathrm{a}$ & $19,75 \mathrm{a}$ & $73,29 \mathrm{a}$ \\
C2 (D3) & $3.968,14 \mathrm{a}$ & $102,00 \mathrm{ab}$ & $12,00 \mathrm{a}$ & $28,60 \mathrm{a}$ & $9,73 \mathrm{a}$ & $17,83 \mathrm{ab}$ & $56,16 \mathrm{ab}$ \\
C2 (D4) & $3.886,27 \mathrm{a}$ & $62,00 \mathrm{ab}$ & $12,00 \mathrm{a}$ & $28,04 \mathrm{a}$ & $9,75 \mathrm{a}$ & $11,26 \mathrm{ab}$ & $49,05 \mathrm{abc}$ \\
\hline Média geral & $\mathbf{3 . 2 6 7 , 9}$ & $\mathbf{9 1 , 2 0}$ & $\mathbf{1 0 , 0 2}$ & $\mathbf{2 4 , 0 8}$ & $\mathbf{8 , 7 0}$ & $\mathbf{1 4 , 4 3}$ & $\mathbf{4 7 , 2 2}$ \\
\hline CV (\%) & $\mathbf{2 9 , 0 1}$ & $\mathbf{4 9 , 6 2}$ & $\mathbf{1 6 , 1 1}$ & $\mathbf{3 4 , 2 3}$ & $\mathbf{3 2 , 8 3}$ & $\mathbf{4 9 , 0 7}$ & $\mathbf{3 7 , 2 5}$ \\
\hline
\end{tabular}

(1) Médias seguidas pela mesma letra nas colunas não diferem estatisticamente, pelo teste de Tukey, a 5\% de probabilidade.

(2) $\mathrm{C} 1$ : composto orgânico preparado pela mistura entre esterco bovino curtido e capim-elefante [0 (D0); 0,853 (D1); 1,706 (D2); 2,559 (D3) e 3,412 (D4) kg por vaso]; C2: composto orgânico preparado pela mistura entre cama-de-frango e capim-elefante [0 (D0); 0,758 (D1); 1,516 (D2); 2,274 (D3) e 3,032 (D4) kg por vaso]. AF: área foliar; VR: volume do sistema radicular; NRP: número de ramos plagiotrópicos; MSF: massa da matéria seca das folhas; MSR: massa da matéria seca dos ramos (ortotrópico + plagiotrópicos); MSSR: massa da matéria seca do sistema radicular; MST: massa da matéria seca total. 
(2006) em que plantas deficientes em $\mathrm{N}$ tendem a apresentar maior teor de $\mathrm{Ca}$.

Não foram observadas diferenças entre os tratamentos quanto aos teores foliares de $\mathrm{P}$ e S (Tabela 4), provavelmente decorrente da aplicação na adubação fosfatada do superfosfato simples que contém cerca de $18 \%$ de $\mathrm{P}_{2} \mathrm{O}_{5}$ e 10-12\% de S (Guimarães et al., 1999).

As doses mais elevadas de ambos compostos proporcionaram os maiores teores foliares de $\mathrm{K}$, fato que pode ser atribuído aos teores elevados de $\mathrm{K}$ em ambos compostos.
A média geral do teor foliar de Ca é semelhante à observada por Partelli et al. (2006) em plantas de cafeeiro conilon em cultivo convencional e está dentro da faixa recomendada por Bragança et al. (2007), entretanto as médias de todos os tratamentos que receberam o $\mathrm{C} 1$ e do tratamento que recebeu a maior dose do $\mathrm{C} 2$ apresentaram teores foliares abaixo da faixa recomendada.

Quanto aos teores foliares de $\mathrm{Mg}$, foi constatada sensível diferença entre os tratamentos (Tabela 4). As médias gerais obtidas dos teores foliares de $\mathrm{Mg}$ estão abaixo das observadas por Partelli et al. (2006) e por Bragança et al.

Tabela 3: Efeito das doses de dois compostos orgânicos sobre o crescimento das plantas de cafeeiro conilon, aos 120 dias após o plantio em vasos

\begin{tabular}{|c|c|c|c|c|}
\hline $\begin{array}{l}\text { Compostos } \\
\text { orgânicos }^{(1)}\end{array}$ & Equação de regressão (5\%) & $\begin{array}{c}\text { Coeficiente de } \\
\text { determinação } \\
\mathbf{R}^{2}\end{array}$ & $\begin{array}{l}\text { Dose máxima } \\
\text { (kg/vaso) }\end{array}$ & $\begin{array}{c}\text { Valor } \\
\text { máximo }\end{array}$ \\
\hline \multicolumn{5}{|c|}{ Altura (cm) } \\
\hline $\mathrm{C} 1$ & $\hat{\mathrm{Y}}=21,593142+14,342 \mathrm{x}-4,3587 \mathrm{x}^{2}$ & 0,98 & 1,645 & 33,39 \\
\hline $\mathrm{C} 2$ & $\hat{Y}=21,794857+15,678 x-4,4879 x^{2}$ & 0,82 & 1,747 & 35,49 \\
\hline \multicolumn{5}{|c|}{ Diâmetro do ramo ortotrópico (mm) } \\
\hline $\mathrm{C} 1$ & $\hat{\mathrm{Y}}=6,390686+3,3756 \mathrm{x}-1,0622 \mathrm{x}^{2}$ & 0,89 & 1,589 & 9,07 \\
\hline $\mathrm{C} 2$ & $\hat{Y}=6,457029+2,5923 x-0,7367 x^{2}$ & 0,83 & 1,759 & 8,74 \\
\hline \multicolumn{5}{|c|}{$\begin{array}{ll}\text { Número de folhas } \\
\end{array}$} \\
\hline $\mathrm{C} 1$ & $\hat{Y}=38,765714+68,849 x-20,282 x^{2}$ & 0,92 & 1,697 & 97,19 \\
\hline $\mathrm{C} 2$ & $\hat{Y}=40,588571+59,898 x-15,639 x^{2}$ & 0,89 & 1,915 & 97,94 \\
\hline \multicolumn{5}{|c|}{ Área foliar $\left(\mathrm{cm}^{2}\right)$} \\
\hline $\mathrm{C} 1$ & $\hat{\mathrm{Y}}=1.316,987+3.719,1 \mathrm{x}-1.088,8 \mathrm{x}^{2}$ & 0,93 & 1,708 & $4.492,89$ \\
\hline $\mathrm{C} 2$ & $\hat{\mathrm{Y}}=1.220,921+4.101,2 \mathrm{x}-1.106,8 \mathrm{x}^{2}$ & 0,87 & 1,854 & $5.025,69$ \\
\hline \multicolumn{5}{|c|}{$\begin{array}{ll}\text { Volume da raíz }(L) \\
\end{array}$} \\
\hline $\mathrm{C} 1$ & $\hat{Y}=0,056114+0,1015 x-0,0318 x^{2}$ & 0,87 & 1,596 & 0,137 \\
\hline $\mathrm{C} 2$ & $\hat{Y}=0,051886+0,1011 x-0,0328 x^{2}$ & 0,94 & 1,541 & 0,130 \\
\hline \multicolumn{5}{|c|}{ Número de ramos plagiotrópicos } \\
\hline $\mathrm{C} 1$ & $\hat{Y}=6,158571+6,2988 x-1,6983 x^{2}$ & 0,80 & 1,854 & 12,00 \\
\hline $\mathrm{C} 2$ & $\hat{Y}=6,057143+6,4191 x-1,5167 x^{2}$ & 0,94 & 2,116 & 12,85 \\
\hline \multicolumn{5}{|c|}{ Matéria seca das folhas (g) } \\
\hline $\mathrm{C} 1$ & $\hat{\mathrm{Y}}=9,697771+27,70 \mathrm{x}-8,2458 \mathrm{x}^{2}$ & 0,89 & 1,680 & 32,96 \\
\hline $\mathrm{C} 2$ & $\hat{Y}=9,410229+31,89 x-8,8594 x^{2}$ & 0,84 & 1,800 & 38,11 \\
\hline \multicolumn{5}{|c|}{ Matéria seca dos ramos $(\mathrm{g})$} \\
\hline $\mathrm{C} 1$ & $\hat{Y}=4,201086+9,3414 x-2,8324 x^{2}$ & 0,88 & 1,649 & 11,90 \\
\hline $\mathrm{C} 2$ & $\hat{Y}=4,013314+10,336 x-2,9155 x^{2}$ & 0,80 & 1,773 & 13,17 \\
\hline \multicolumn{5}{|c|}{ Matéria seca do sistema radicular $(\mathrm{g})$} \\
\hline $\mathrm{C} 1$ & $\hat{Y}=10,781371+11,826 x-3,9756 x^{2}$ & 0,86 & 1,487 & 19,58 \\
\hline $\mathrm{C} 2$ & $\hat{Y}=8,8094860+15,097 x-4,7668 x^{2}$ & 0,95 & 1,584 & 20,76 \\
\hline \multicolumn{5}{|c|}{ Matéria seca total (g) } \\
\hline $\mathrm{C} 1$ & $\hat{Y}=24,680229+48,867 x-15,054 x^{2}$ & 0,89 & 1,623 & 64,34 \\
\hline $\mathrm{C} 2$ & $\hat{Y}=22,233030+57,324 x-16,542 x^{2}$ & 0,87 & 1,733 & 71,90 \\
\hline
\end{tabular}

(1) C1: composto orgânico preparado pela mistura entre esterco bovino curtido e capim-elefante (1:4); C2: composto orgânico preparado pela mistura entre cama-de-frango e capim-elefante (1:4). 
(2007), entretanto não foram observados sintomas visuais de deficiência deste nutriente nas plantas.

Os baixos teores foliares de $\mathrm{Ca}$ e $\mathrm{Mg}$ observados podem ser devido aos elevados teores de K nos compostos, pois existe competição na absorção destes cátions pelas raízes (Malavolta, 2006). Entretanto, segundo este mesmo autor, o $\mathrm{P}$ tende a diminuir o efeito depressivo do $\mathrm{K}$ no teor foliar de $\mathrm{Mg}$.

Ao analisar-se o efeito de cada composto orgânico avaliado e de suas respectivas doses, verifica-se que o $\mathrm{C} 1$ não proporcionou diferença entre as doses aplicadas em relação aos teores de foliares de $\mathrm{P}, \mathrm{Mg}$ e $\mathrm{S}$, e o C2 não influenciou de forma significativa os teores de K, Mg e S (Tabela 5).
Para ambos os compostos, foram observadas respostas quadráticas nos teores foliares de N (Tabela 5), sendo que os maiores teores foliares foram alcançados com a aplicação de 2,389 kg/vaso de C1 e 2,374 kg/vaso de C2.

$\mathrm{O}$ aumento das doses do $\mathrm{C} 1$ e do $\mathrm{C} 2$ promoveu o aumento linear nos teores foliares de $\mathrm{K} \mathrm{e} \mathrm{P}$, respectivamente (Tabela 5), e um decréscimo nos teores foliares de $\mathrm{Ca}$, fato que pode ser explicado pelo aumento da aplicação de K (Malavolta, 2006).

Com os resultados obtidos, pode-se inferir que a adubação com compostos orgânicos no plantio pode ser uma alternativa para substituir ou reduzir as adubações minerais nitrogenada e potássica recomendadas para a fase de pós-plantio das mudas do cafeeiro conilon.

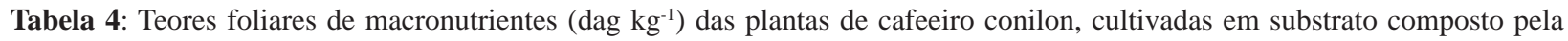
mistura de solo e diferentes doses de dois compostos orgânicos, aos 120 dias após o plantio em vasos ${ }^{(1)}$

\begin{tabular}{lcccccc}
\hline Tratamentos $^{(2)}$ & $\mathbf{N}$ & $\mathbf{P}$ & $\mathbf{K}$ & $\mathbf{C a}$ & $\mathbf{M g}$ & $\mathbf{S}$ \\
\hline C1 (D0) & $1,78 \mathrm{c}$ & $0,19 \mathrm{a}$ & $2,88 \mathrm{~b}$ & $1,65 \mathrm{~b}$ & $0,17 \mathrm{ab}$ & $0,53 \mathrm{a}$ \\
C1 (D1) & $3,04 \mathrm{~b}$ & $0,14 \mathrm{a}$ & $3,06 \mathrm{~b}$ & $0,95 \mathrm{c}$ & $0,18 \mathrm{ab}$ & $0,24 \mathrm{a}$ \\
C1 (D2) & $3,79 \mathrm{a}$ & $0,17 \mathrm{a}$ & $3,58 \mathrm{ab}$ & $0,95 \mathrm{c}$ & $0,18 \mathrm{ab}$ & $0,30 \mathrm{a}$ \\
C1 (D3) & $3,95 \mathrm{a}$ & $0,18 \mathrm{a}$ & $4,30 \mathrm{a}$ & $0,87 \mathrm{c}$ & $0,16 \mathrm{ab}$ & $0,32 \mathrm{a}$ \\
C1 (D4) & $3,56 \mathrm{ab}$ & $0,17 \mathrm{a}$ & $4,21 \mathrm{a}$ & $0,77 \mathrm{c}$ & $0,14 \mathrm{~b}$ & $0,24 \mathrm{a}$ \\
C2 (D0) & $2,02 \mathrm{c}$ & $0,15 \mathrm{a}$ & $2,91 \mathrm{~b}$ & $2,12 \mathrm{a}$ & $0,17 \mathrm{ab}$ & $0,33 \mathrm{a}$ \\
C2 (D1) & $3,12 \mathrm{~b}$ & $0,16 \mathrm{a}$ & $3,15 \mathrm{~b}$ & $1,13 \mathrm{c}$ & $0,14 \mathrm{~b}$ & $0,27 \mathrm{a}$ \\
C2 (D2) & $3,95 \mathrm{a}$ & $0,17 \mathrm{a}$ & $3,00 \mathrm{~b}$ & $1,04 \mathrm{c}$ & $0,17 \mathrm{ab}$ & $0,27 \mathrm{a}$ \\
C2 (D3) & $3,95 \mathrm{a}$ & $0,18 \mathrm{a}$ & $3,32 \mathrm{ab}$ & $1,12 \mathrm{c}$ & $0,19 \mathrm{a}$ & $0,28 \mathrm{a}$ \\
C2 (D4) & $3,99 \mathrm{a}$ & $0,19 \mathrm{a}$ & $3,55 \mathrm{ab}$ & $0,91 \mathrm{c}$ & $0,17 \mathrm{ab}$ & $0,31 \mathrm{a}$ \\
\hline Média geral & $\mathbf{3 , 3 1}$ & $\mathbf{0 , 1 7}$ & $\mathbf{3 , 4 0}$ & $\mathbf{1 , 1 5}$ & $\mathbf{0 , 1 7}$ & $\mathbf{0 , 3 1}$ \\
\hline CV (\%) & $\mathbf{9 , 2 5}$ & $\mathbf{2 1 , 5 2}$ & $\mathbf{1 3 , 8 8}$ & $\mathbf{1 5 , 5 9}$ & $\mathbf{1 4 , 8 0}$ & $\mathbf{4 6 , 9 8}$ \\
\hline
\end{tabular}

(1) Médias seguidas pela mesma letra nas colunas não diferem estatisticamente, pelo teste de Tukey, a 5\% de probabilidade.

(2) $\mathrm{C} 1$ : composto orgânico preparado pela mistura entre esterco bovino curtido e capim-elefante [0 (D0); 0,853 (D1); 1,706 (D2); 2,559 (D3) e 3,412 (D4) kg por vaso]; C2: composto orgânico preparado pela mistura entre cama-de-frango e capim-elefante [0 (D0); 0,758 (D1); 1,516 (D2); 2,274 (D3) e 3,032 (D4) kg por vaso].

Tabela 5: Efeito das doses de dois compostos orgânicos sobre os teores foliares de macronutrientes das plantas de cafeeiro conilon, aos 120 dias após o plantio em vasos

\begin{tabular}{|c|c|c|c|c|}
\hline $\begin{array}{l}\text { Compostos } \\
\text { orgânicos }^{(1)}\end{array}$ & Equação de regressão (5\%) & $\begin{array}{c}\text { Coeficiente de } \\
\text { determinação } \\
\mathbf{R}^{2}\end{array}$ & $\begin{array}{c}\text { Dose máxima } \\
\text { (kg/vaso) }\end{array}$ & $\begin{array}{c}\text { Valor } \\
\text { máximo }\end{array}$ \\
\hline \multicolumn{5}{|c|}{$\mathbf{N}\left(\right.$ dag kg $\left.{ }^{-1}\right)$} \\
\hline $\mathrm{C} 1$ & $\hat{Y}=1,771600+1,8319 x-0,3834 x^{2}$ & 0,99 & 2,389 & 3,96 \\
\hline $\mathrm{C} 2$ & $\hat{Y}=2,028000+1,7433 x-0,3672 x^{2}$ & 0,99 & 2,374 & 4,10 \\
\hline \multicolumn{5}{|c|}{$P\left(\right.$ dag kg $\left.^{-1}\right)$} \\
\hline $\mathrm{C} 2$ & $\hat{\mathrm{Y}}=0,15600+0,0185 \mathrm{x}$ & 0,72 & 3,032 & 0,21 \\
\hline \multicolumn{5}{|c|}{ K $\left(\right.$ dag kg $\left.^{-1}\right)$} \\
\hline $\mathrm{C} 1$ & $\hat{\mathrm{Y}}=2,826000+0,4572 \mathrm{x}$ & 0,91 & 3,412 & 4,38 \\
\hline \multicolumn{5}{|c|}{$\mathrm{Ca}\left(\right.$ dag kg-1) $^{-1}$} \\
\hline $\mathrm{C} 1$ & $\hat{Y}=1,405200-0,2157 x$ & 0,70 & 0,00 & 1,40 \\
\hline $\mathrm{C} 2$ & $\hat{Y}=1,752800-0,3222 x$ & 0,62 & 0,00 & 1,75 \\
\hline
\end{tabular}

(1) C1: composto orgânico preparado pela mistura entre esterco bovino curtido e capim-elefante (1:4); C2: composto orgânico preparado pela mistura entre cama-de-frango e capim-elefante $(1: 4)$.

Rev. Ceres, Viçosa, v. 58, n.1, p. 100-107, jan/fev, 2011 


\section{CONCLUSÕES}

Os compostos orgânicos preparados pelas misturas de esterco bovino curtido e capim-elefante (C1), e de camade-frango e capim-elefante (C2), na proporção 1:4, nas quantidades aplicadas, influenciam o crescimento e o estado nutricional das plantas de cafeeiro conilon;

Os valores máximos de altura das plantas, diâmetro de caule e matéria seca total são obtidos com as doses de 1,$645 ; 1,589$ e 1,623 kg/vaso do C1 (entre 7,945 a 8,225 kg/ cova), e 1,747; 1,759 e 1,733 kg/vaso do C2 (entre 8,665 a $8,795 \mathrm{~kg} /$ cova), respectivamente;

Nestas mesmas doses, as plantas apresentam teores foliares de N e K acima da faixa de suficiência;

A adubação com compostos orgânicos no plantio pode ser uma alternativa para substituir ou reduzir as adubações nitrogenada e potássica recomendadas para a fase de pós-plantio do cafeeiro conilon.

\section{AGRADECIMENTOS}

Ao Banco do Nordeste do Brasil S/A, pelo apoio financeiro; ao INCAPER pelo apoio estrutural e ao Biólogo Alex Fabian Rabelo Teixeira (INCAPER), pela ajuda na realização do experimento.

\section{REFERÊNCIAS}

ANDA - Associação Nacional para Difusão de Adubos. (2009) Disponível em: <http://www.anda.org.br/home.aspx>. Acessado em: 16 de fevereiro de 2009.

Andrade Neto A, Mendes ANG \& Guimarães PTG (1999) Avaliação de substratos alternativos e tipos de adubação para a produção de mudas de cafeeiro (Coffea arabica L.) em tubetes. Ciência e Agrotecnologia, 23:270-280.

Bragança SM, Martinez, HEP, Garcia, H, Santos, LP, Sediyama, CS, Alvarez Venegas, VH \& Lani, JA (2010) Acumulação de matéria seca pelo cafeeiro conilon. Revista Ceres, 57: 48-52.

Bragança SM, Prezotti LC \& Lani JA (2007) Nutrição do cafeeiro conilon. In: Ferrão, R. G. et al. (eds) Café Conilon. Vitória, Incaper, p.299-327.

Cervellini GS, Campana MP, Igue T \& Toledo SV (1995) Modo de aplicação de esterco e de fertilizantes minerais no cafeeiro. Bragantia, 54:169-176.

Cervellini GS \& Igue T (1994) Adubação mineral e orgânica do cafeeiro. Bragantia, 53:83-93.

CONAB - Companhia Nacional de Abastecimento (2010) Acompanhamento da safra brasileira de café Safra 2010, quarta estimativa, dezembro/2010. Disponível em: 〈http://www.conab.gov.br>. Acessado em: 20/12/2010.

Dias R \& Melo B (2009) Proporção de material orgânico no substrato artificial para a produção de mudas de cafeeiro em tubetes. Ciência e Agrotecnologia, 33:144-152.

Guimarães PTG, Garcia AWR, Alvarez Viegas VH, Prezotti LC, Viana AS, Miguel AE, Malavolta, E Corrêa, JB, Lopes, AS, Nogueira, FD \& Monteiro, AVC (1999). Cafeeiro. In: Ribeiro, AC; Guimarães, PTG \& Alvarez Viegas, VH (Ed.). Recomendações para o uso de corretivos e fertilizantes em Minas Gerais: $5^{\text {a }}$ aproximação. Viçosa: Comissão de Fertilidade do Solo do Estado de Minas Gerais. p.289-302.
Malavolta E (2006) Manual de nutrição mineral de plantas. São Paulo: Editora Agronômica Ceres, 638p.

Marana JP, Miglioranza E, Fonseca EP \& Kainuma RH (2008) Índices de qualidade e crescimento de mudas de café produzidas em tubetes. Ciência Rural, 38:39-45.

Partelli FL, Vieira, HD, Monnerat, PH \& Viana, AP (2006) Estabelecimento de normas Dris em cafeeiro conilon orgânico ou convencional no estado do Espírito Santo. Revista Brasileira de Ciência do Solo, 30: 443-451.

Pozza AAA, Martinez, HEP, Caixeta, SL, Cardoso, AA, Zambolim, L \& Pozza, EA (2001) Influência da nutrição mineral na intensidade da mancha-de-olho pardo em mudas de cafeeiro. Pesquisa Agropecuária Brasileira, 36: 53-60.

Prezotti LC, Bragança SM, Guarçoni-M A \& Lani JA (2007) Calagem e adubação do cafeeiro conilon. In: Ferrão, R. G. et al. (eds) Café Conilon. Vitória, Incaper, p.329-343. 